Journal of Animal and Veterinary Advances 18 (2): 48-54, 2019

ISSN: $1680-5593$

(C) Medwell Journals, 2019

\title{
Using Landmark and Semi-Landmark-Based Geometric Morphometric to Identify Three Species of Aedes Mosquitoes in Thailand
}

\author{
Tanawat Chaiphongpachara and Sedthapong Laojun \\ Department of Public Health, College of Allied Health Sciences, Suan Sunandha Rajabhat University, \\ Samut Songkhram, Thailand
}

\begin{abstract}
Geometric Morphometrics (GM) is a new effective technique for mosquito identification and has become popular in applications to entomological work. In this study, we evaluated the Landmark and Semi-Landmark-based approach (LMSL) of GM to distinguish three species of Aedes mosquitoes in Thailand, including Aedes aegypti, Ae. albopictus and Ae. scutellaris as medically important vectors and compared the efficacy of LMSL and the Landmark-based approach (LM) to investigate both of their potentials. We were collected Aedes populations at the larval and pupal stages in human-made water containers in the communities of the Mueang Samut Songkhram District, Samut Songkhram Province, Thailand. The results of the comparison between LM and LMSL in this study revealed that they had similar efficacies for three Aedes species separations based on shape variation. However, for some Aedes species such as Ae. scutellaris, LMSL can better separate than LM while Ae. albopictus have better separation with LM than LMSL. Therefore, we recommend that before applying this technique, there should be a comparison with other GM methods to determine the specificity of species of mosquito vector in order to find an appropriate GM technique.
\end{abstract}

Key words: Geometric morphometric, landmark and semi-landmark-based approach, Aedes aegypti, Aedes albopictus, Aedes scutellaris, GM methods

\section{INTRODUCTION}

Dengue is still a major problem causing illness and death worldwide, especially in tropical and sub-tropical areas (Chaiphongpachara et al., 2017; Lee et al., 2018). Over the last 50 years, dengue's incidence has risen 30-fold over with an estimated 50-100 million infections per year and more than 100 endemic countries (Guzman et al., 2010; Ebi and Nealon, 2016). Chikungunya is also a major public health problem that mostly occurs in Africa, Asia and the Indian subcontinent (Mahendradas, 2016; Vu et al., 2017). Both dengue and chikungunya are mosquito-borne viral diseases transmitted by Aedes mosquitoes (Service, 2008; Chaiphongpachara and Moolrat, 2017). Aedes aegypti, Ae. albopictus and Ae. scutellaris belong to a subgenus of Stegomyia (Diptera, Culicidae) which are vectors associated with these diseases (Sumruayphol et al., 2016). Typically, these three species of Aedes mosquitoes are of the sympatric field. However, they have unique characteristics, including patterns on the thorax such as lyre-shaped white markings for Ae. aegypti and a long median-longitudinal white stripe for Ae. albopictus but often fail to distinguish species owing to damage with the Aedes specimen, especially, the scutum part (Rattanarithikul et al., 2010). These classification errors may have led to difficulties in planning for Aedes mosquito population control in endemic areas. New alternative methods to resolve this problem are needed.

Geometric Morphometric (GM) is a novel, effective technique based on the coordinates of identifiable landmarks that has become popular in applications to entomological work, especially insects of the family, Culicidae (Lorenz et al., 2012, 2017; Chaiphongpachara, 2018). The advantages of this technique are that its inexpensive, easy to use and fast, supported by specialized analysis software (Garros and Dujardin, 2013; Dujardin, 2008). Therefore, this procedure is so attractive that even though molecular techniques are powerful butthey are expensive. Many species of mosquitoes have numerous similarities in terms of morphology such as species complex, cryptic species, sibling species and morphologically close species, so, sometimes, morphological identification that occurs is faulty (WHO.,

Corresponding Author: Tanawat Chaiphongpachara, Department of Public Health, College of Allied Health Science, Suan Sunandha Rajabhat University, Samut Songkhram, Thailand 
2007). The Landmark-based approach (LM) is the first geometric morphometric method to solve the mosquito classification problem. Wings of mosquitoes are suitable organsforanalysis by GM techniques which are bi-dimensional and specific to each species (Wilke et al., 2016). However, the weaknesses of the LM method include that it may not cover certain landmarks at curved positions, unique features of certain species. For mosquitoes in some areas of the wing, there is an important curve that may be species-specific.

The Semi-Landmark-based (LMSL) approach is a GM technique developed from LM combined with semi-landmarks (locations of points on curves) analyses (Kaba et al., 2017). Therefore, in this study, we assessed the LM and LMSL approach of GM to distinguish three species of Aedes mosquitoes in Thailand, including Aedes aegypti, Ae. albopictus and Ae. scutellaris-medically importance vectors. In addition, we were compared the efficacy of LMSL and LM to investigate the potential of both approaches for identifying Aedes mosquitoes.

\section{MATERIALS AND METHODS}

Aedes collection and identification: In this study, we collected Aedes populations at the larval and pupal stages in human-made water containers usinga dipper, ladle spoon, dropper and pipette. All sample collection was carried out in the communities of the Mueang Samut Songkhram District, Samut Songkhram Province, Thailand during August 2018. Afterwards, mosquito samples were sent to the College of Allied Health Sciences, Suan Sunandha Rajabhat University for rearing in a laboratory. Controlling the rearing conditions in laboratory was essential and in this study was set as $25 \pm 2^{\circ} \mathrm{C}, 50-60 \%$ relative humidity and 12:12 h light: dark. Larvae and pupae were reared in plastic trays containing filtered water $(30 \times 40 \times 7 \mathrm{~cm})$ and were provided $0.1 \mathrm{~g}$ of dog food daily. After emergence, pupae were transferred to $30 \times 30 \times 30 \mathrm{~cm}$ cagesto wait for the development of the adult stage. Only adult female Aedes mosquitoes as vectors (male mosquitoes are not vectors) were identified based on the morphological taxonomic keys (Rattanarithikul et al., 2005, 2010).

Wing preparation and data collection: Female adult Aedes aegypti, Ae. albopictus and Ae. scutellaris were used in this study. The samples employed were selected from the integrity of the wings without tearing and clear visibility of wing venation. The right wing of each Aedes sample was dissected and mounted on microscope slides with a coverslip using Hoyer's medium. Thereafter, Aedes wings on slides were photographed by a Nikon DS-Ril SIGHT digital camera connected to a Nikon Eclipse E600 microscope (Nikon Corp., Tokyo, Japan) at $40 \times$ magnification with a scalebar $(1 \mathrm{~mm})$ indicated on the photograph.

\section{Geometric morphometric analyses}

Landmark-based GM approach (LM): The 11 landmarks in Fig. 1 were digitized and used for LM in this study. After landmark collection, all photographs were repeated and compared to the set used for investigating the quality of landmarks before GM analysis (repeatability test) (Arnqvist and Martensson, 1998).

Size variations between Aedes species was estimated by Centroid Size (CS) which is the square root of the sum of the squared distances between the center of the configuration of landmarks and each landmark (Bookstein, 1991). CS variation was created as quantile boxes for comparison between Aedes groups while significant differences for CS variation among Aedes mosquitoes was based on non-parametric, permutation tests (1000 cycles) with post hoc pair wise Bonferroni correction testat $\mathrm{p}<0.05$.

Shape variable (Partial Warp (PW) scores) of Aedes wings were computed from principal components variables from principal components was performed to calculated after generalized Procrustes analysis.
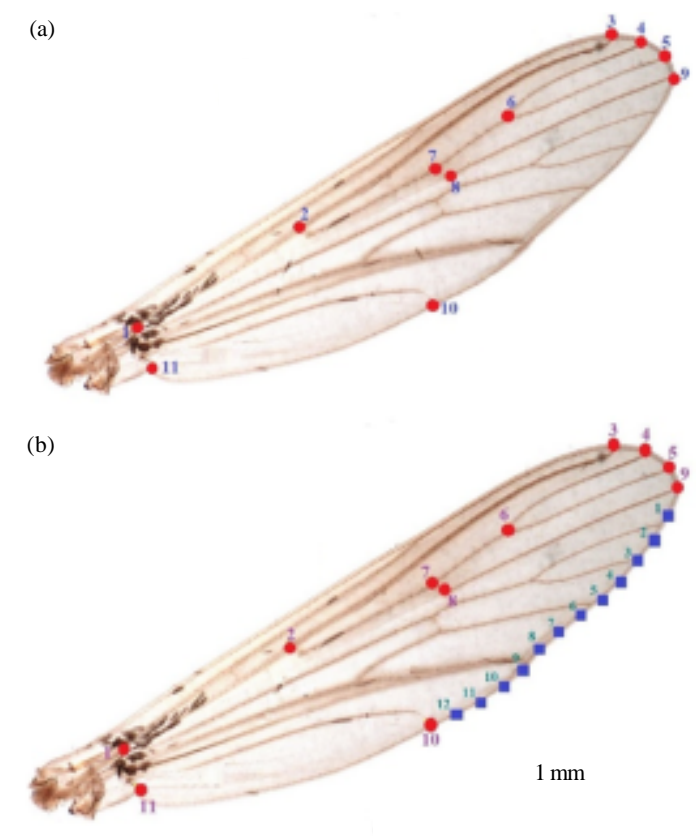

Fig. 1: a) Positions of 11 Landmarks for LM and b) 11 landmarks combined with 12 semi-landmarks for LMSL on Aedes wings 
Meanwhile discriminant analysis of the canonical create a factor map. Mahalanobis distances were calculated from discriminant analysis to estimate the distance between species. The statistical significance of pairwise Mahalanobis distances between Aedes species was computed by non-parametric, permutation tests (1000 cycles) with Bonferroni correction at $\mathrm{p}<0.05$. The accuracy of Aedes species classification was based on wing shape and each individual was reclassified by comparing wing similarity to the mean shape of each species based in terms of Mahalanobis distances comparisons (cross-validated classification test). A neighbor-joining tree was constructed based on Procrustes distances to determine the similarity of the shape in each Aedes species and added Culex quinquefasciatus for a putative outgroup.

LM and LMSL-based approach: For the LM and LMSL-based approaches, there were very similar analytical processes including repeatability test, CS analysis, shape analysis, cross-validated classification, significance test and neighbor-joining tree. However, it has a different element which is the landmark collection. Determining the point of this method is the combination of the 11 Landmarks of LM and the 12 semi-landmarks at the curved line of the vein between landmarks 9 and 10 of the lower angle of the mosquito wing (Fig. 1).

Software: Analyses and computation by both GM approaches in this research made use of specialized software, including CLIC (Collection of Landmarks for Identification and Characterization), package Version 97 (Dujardin, 2011) which is freely available at https://xyom-clic.eu. Five modules of CLIC Software were utilized for GM analyses, including $\mathrm{COO}$ for digitizing 11 landmarks and 12 semi-landmarks, TET to edit, convert and compute data, MOG to generate centroid size and shape variables, formulate quantile box of size variation and compute Procrustes distances, VAR for analyze size variation and testing statistical difference of size between species and PAD for analysis of shape variation, permutation-based statistical significance of Mahalanobis distances between species and cross-validated reclassifications. Finally, R Software (https://cran.r-project.org/) was applied to build neighborjoining trees for LM and LMSL.

\section{RESULTS AND DISCUSSION}

The 45 wings of Ae. aegypti, 34 wings of Ae. albopictus and 34 wings of Ae. scutellaris were used which was the same set for analysis of LM and LMSL.
Before analysis, we repeatedly measured photographs and found that the photograph set used for the analysis had robust quality for pointing landmarks (LM: repeatability size was 0.94 and for shape it was 0.96 ; LMSM; repeatability of size was 0.92 and shape was $0.94)$.

Size analyses: CS were used for wing size estimation in both LM and LMSL. Variations of CS are portrayed asquantile boxes for comparison between Aedes species in Fig. 2. The CS average indicates that Ae. aegypti had the largest wings followed by Ae. scutellaris and Ae. albopictus in LM while Ae. aegypti had the largest wings followed by Ae. albopictus and Ae. scutellaris in LMSL (Table 1). The statistically significant difference test found that the both LM and LMSL approaches had varying results for $\mathrm{CS}$ between species. In LM, $A e$. albopictus was different from other species while $A e$. scutellaris was different from other species with LMSL (Table 1).

Shape analysis: After generalized Procrustes analysis, shape variation between species was determined byconnecting mean landmark configuration of 11 Landmarks for LM and 11 landmarks combined with 12 semi-landmarks for LMSL by a straight line (Fig. 3). Comparison among the three Aedes species has been shown as a factor map for both LM and LMSL as shown in Fig. 4. With the comparison of wing shape between species, we found permutation-based statistically significant differences in Mahalanobis distances for all Aedes species with both approaches $(\mathrm{p}<0.05$, Table 2 and 3$)$.

The evaluation of the accuracy of classification of both LM and LMSL (cross-validated classification) showed that LM provided Aedes species separation similar to LMSL. The range of cross-validated classification scores based on shape variation in LM was $55-86 \%$ while the range for LMSL was $50-86 \%$ of which Ae. aegypti had the most cross-validated classification scores with both approaches. The similarity of shape for each species of both LM and LMSL was calculated and constructed as shown in the neighbor-joining trees in Fig. 5.

In the research presented here, we evaluated and compared the performance of female Aedes mosquito separation, including those from the species Aedes aegypti, Ae. albopictus and Ae. scutellaris, via. LM and LMSL. Analysis of these techniques can be divided into two parts-size and shape. However, with the species identification of mosquitoes, the focus is usually on wing shape, though to investigate the potential of both methods, we had to analyze wing size. 

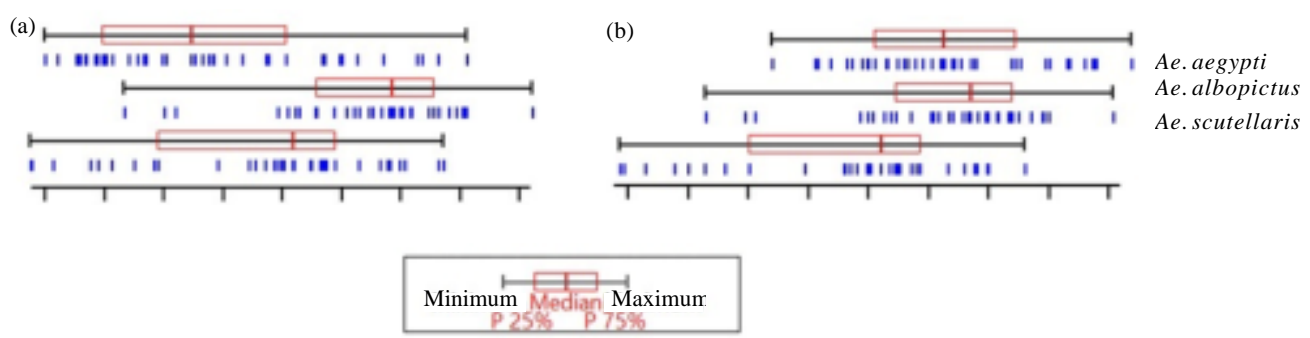

Fig. 2: CS variation of the wings between Aedes species (units are $\mathrm{mm}$ ) shown as quantile boxes: a) SL and b) LMSL). Each box features the median separating the 25 and $75 \%$ quartiles

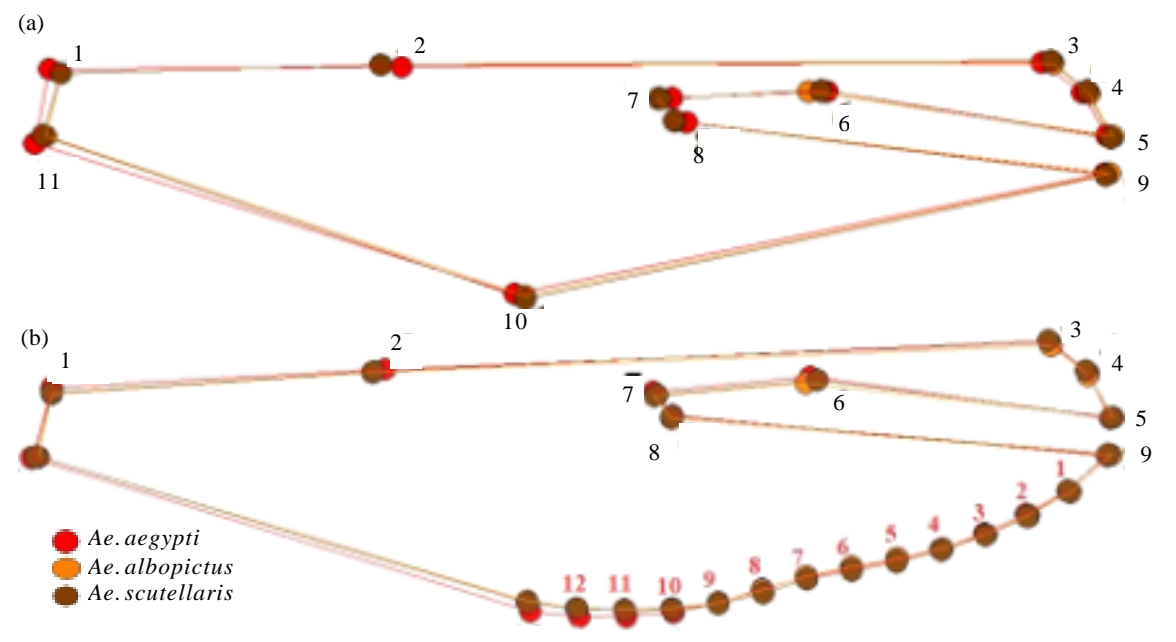

Fig. 3: Superimposition of the mean landmark configurations of Ae.aegypti(red), Ae. albopictus (orange) and Ae. scutellaris (brown): a) LM and b) LMSL

Table 1: Statistical comparisons of wing CS between species in LM and LMSL

\begin{tabular}{lll}
\hline & Mean \pm SD of wing CS (mm) \\
\cline { 2 - 3 } Species & LM & LMSL \\
\hline Ae. aegypti & $2.55 \pm 0.10^{\mathrm{a}}$ & $3.29 \pm 0.08^{\mathrm{a}}$ \\
Ae. albopictus & $2.88 \pm 0.06^{\mathrm{b}}$ & $3.33 \pm 0.07^{\mathrm{a}}$ \\
Ae. scutellaris & $2.62 \pm 0.09^{\mathrm{a}}$ & $3.02 \pm 0.12^{\mathrm{b}}$ \\
\hline a, ${ }^{\mathrm{b}}$ Different superscript letters in rows represent statistical differences at \\
p<0.05 (mm: milli meters; mean: average of centroid size; SD: Standard \\
Deviation)
\end{tabular}

Both LM and LMSL have similar wing size based on $\mathrm{CS}$ averaging. However, the permutation-based statistical tests show different CS averages for each species which were different patterns between LM and LMSL, including Ae. albopictus that was different from other species in LM while Ae. scutellaris was different from other species with LMSL. As such it may be based on the different number of landmark points that affect the results of the size analysis. In addition, varied approaches also affect size
Table 2: Statistical significance of pairwise Mahalanobis distances between species in LM and LMSL

\begin{tabular}{lccc}
\hline Approaches/species & Ae. aegypti & Ae. albopictus & Ae. Scutellaris \\
\hline LM & & & \\
Ae. aegypti & - & & \\
Ae. albopictus & $3.25^{*}$ & - & - \\
Ae. scutellaris & $3.28^{*}$ & $1.75^{*}$ & - \\
LMSL & & & \\
Ae. aegypti & - & - & \\
Ae. albopictus & $4.59^{*}$ & $3.15^{*}$ & - \\
Ae. Scutellaris & $5.03^{*}$ &
\end{tabular}

analysis. This was consistent with the previous results that compared the findings with trombiculid mites within the genus, Walchia in terms of identification of LM and an outline-based GM method that yielded different size patterns between both approaches (Sungvornyothin et al., 2019).

Wing shape is important factor for mosquito species identification using GM. The wing is the organ that 

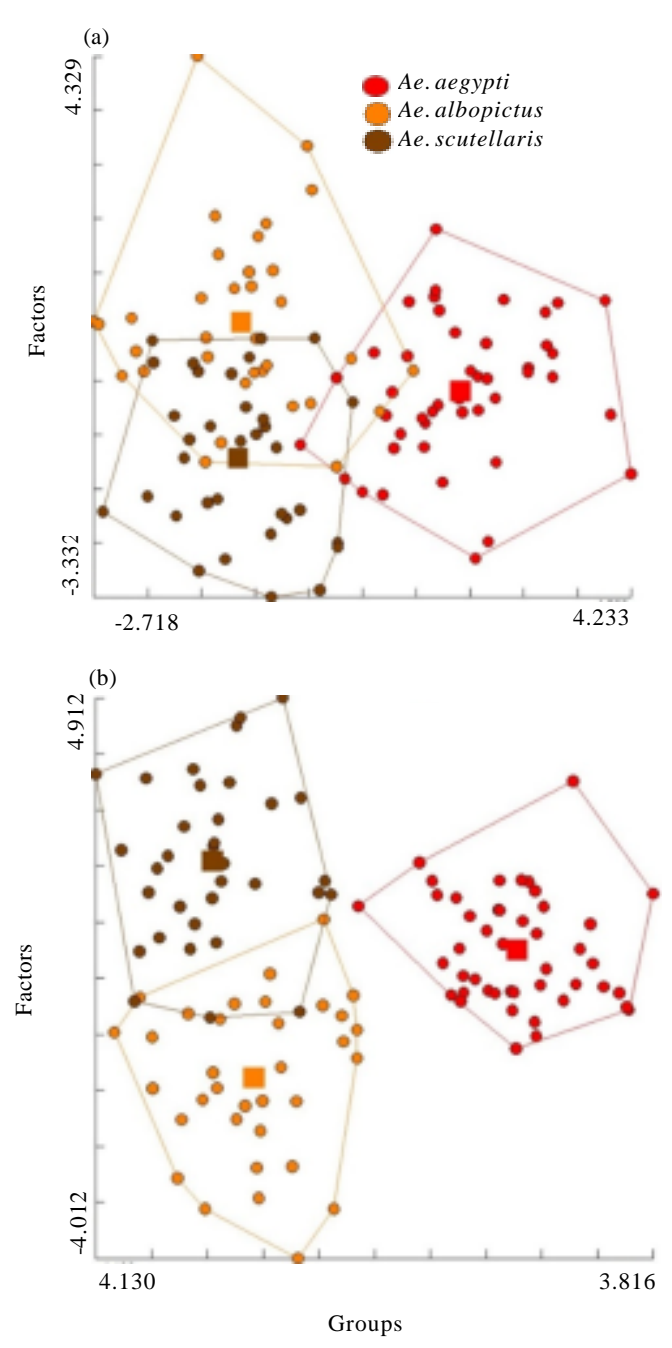

Fig. 4: Factor maps for discriminant analysis resultant from comparison among the three Aedes species, including Ae. aegypti (red), Ae. albopictus (orange) and Ae. scutellaris (brown): a) LM and b) LMSL. Each point represented an individual Aedes for each group

Table 3: Cross-validated classification of Aedes species in LM and LMSL Accuracy of classification (assigned/observed)

\begin{tabular}{lll} 
& & \\
Species & LM (\%) & LMSL (\%) \\
Ae. aegypti & $86(39 / 45)$ & $86(39 / 45)$ \\
Ae. albopictus & $55(19 / 34)$ & $50(17 / 34)$ \\
Ae. scutelloris & $61(21 / 34)$ & $67(23 / 34)$ \\
\hline
\end{tabular}

displays the most unique identity when exploring intraspecific variations among mosquito populations and differences in the wings are derived from sex (Virginio et al., 2015), geographical location (Demari-Silva et al., 2014), phylogenetic relationships (Sumruayphol et al., 2016), ecological relationships

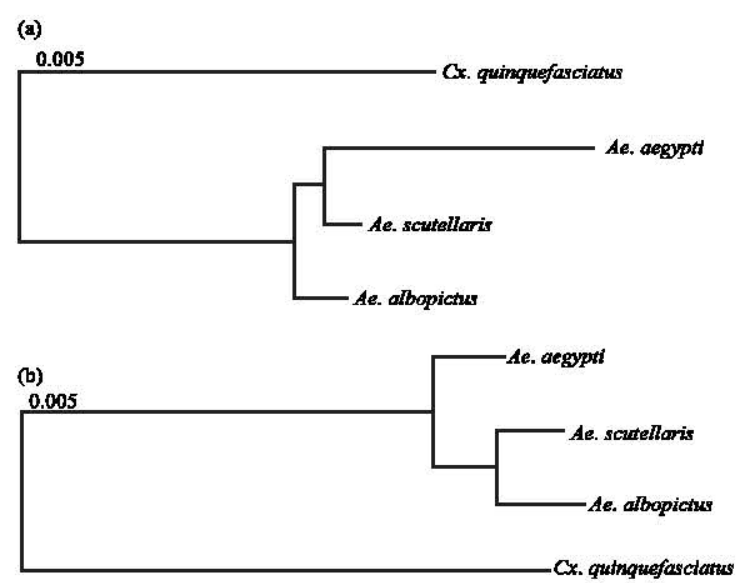

Fig. 5: Neighbor-joining trees based on Procrustes distances between Aedes species: a) LM and b) LMSL

(Hidalgo et al., 2015) and types of treatment (Lorenz et al., 2017). While size is a factor that easily changes based on effects of the environment and food received it is unsuitable for species identification (Dujardin, 2011). The results of shape analyses with LMSL indicate the efficacy of Aedes identification based on discriminant analysis and permutation-based statistically significant differences in Mahalanobis distances. In addition, comparison of cross-validated classification scores of LMSL with LM as a popular approachyielded that they had similar efficacies for three Aedes species separation. However, in certain Aedes species such as Ae. scutellaris, LMSL can better separate than LM while in other species such as Ae. albopictus, LM can better separate than LMSL. In line with earlier research it has been found that LMSL can better identify tsetse fly species than LM. The close relationship of shape between the species expressed as a neighbor-joining tree meant we observed the difference between the approaches caused by 12 semi-landmarks on the LMSL curve.

\section{CONCLUSION}

GM is an alternative technique that has become popular for species identification with applications to entomological work. LMSL is one GM technique that has a strong fit for curved positions whereas other GM methods are difficult, like LM. The results of the comparison between LM and LMSL in this study have revealed that they had similar efficacies for three Aedes species separation, including Aedes aegypti, Ae. albopictus and Ae. scutellaris, based on shape variation. However, for certain Aedes species such as 
Ae. scutellaris, LMSL can better separate than LM while for other species, like Ae. albopictus, LM can better separate than LMSL.

\section{RECOMMENDATIONS}

Therefore, we recommend that before applying this technique, there should be a comparison to other methods to determine the specificity of species of mosquito vector when seeking an appropriate GM technique.

\section{ACKNOWLEDGEMENT}

We would like to express our grateful thanks to the College of Allied Health Sciences, Suan Sunandha Rajabhat University, Thailand for its support of this study.

\section{REFERENCES}

Arnqvist, G. and T. Martensson, 1998. Measurement error in geometric morphometrics: Empirical strategies to assess and reduce its impact on measures of shape. Acta Zool. Academiae Scientiarum Hungaricae, 44: 73-96.

Bookstein, F.L., 1991. Morphometric Tools for Landmark Data: Geometry and Biology. Cambridge University Press, Cambridge, London.

Chaiphongpachara, T. and L. Moolrat, 2017. Insecticide resistance of temephos on Aedes aegypti as dengue vector in Samut Songkhram, Thailand. Ann. Trop. Med. Public Health, 10: 1439-1442.

Chaiphongpachara, T., 2018. Comparison of landmark-and outline-based geometric morphometrics for discriminating mosquito vectors in Ratchaburi Province, Thailand. BioMed Res. Intl., 2018: 1-10.

Chaiphongpachara, T., S. Pimsuka, W.S.N. Ayudhaya and W. Wallapa, 2017. The application of geographic information system in dengue haemorrhagic fever risk assessment in Samut Songkhram Province, Thailand. Intl. J. GEOMATE, 12: 53-60.

Demari-Silva, B., L. Suesdek, M.A.M. Sallum and M.T. Marrelli, 2014. Wing geometry of Culex coronator (Diptera: Culicidae) from South and Southeast Brazil. Parasites Vectors, 7: 1-9.

Dujardin, J.P., 2008. Morphometrics applied to medical entomology. Infect. Genet. Evol., 8: 875-890.

Dujardin, J.P., 2011. Modern Morphometrics of Medically Important Insects. In: Genetics and Evolution of Infectious Disease, Michel, T. (Ed.). Elsevier, Amsterdam, Netherlands, ISBN:978-0-12-384890-1, pp: 473-501.
Ebi, K.L. and J. Nealon, 2016. Dengue in a changing climate. Environ. Res., 151: 115-123.

Garros, C. and J.P. Dujardin, 2013. Genetic and Phenetic Approaches to Anopheles Systematics. In: Anopheles Mosquitoes-New Insights into Malaria Vectors, Manguin, S. (Ed.). IntechOpen, England, UK., ISBN:978-953-51-1188-7, pp: 81-105.

Guzman, M.G., S.B. Halstead, H. Artsob, P. Buchy and J. Farrar et al., 2010. Dengue: A continuing global threat. Nat. Rev. Microbiol., 8: 7-16.

Hidalgo, K., J.P. Dujardin, K. Mouline, R.K. Dabire and D. Renault et al., 2015. Seasonal variation in wing size and shape between geographic populations of the malaria vector, Anopheles coluzzii in Burkina Faso (West Africa). Acta Trop., 143: 79-88.

Kaba, D., D. Berte, B.T.D. Ta, J. Telleria and P. Solano et al., 2017. The wing venation patterns to identify single tsetse flies. Infect. Genet. Evol., 47: 132-139.

Lee, H., S. Halverson and N. Ezinwa, 2018. Mosquito-borne diseases. Primary Care Clinics Office Pract., 45: 393-407.

Lorenz, C., F. Almeida, F. Almeida-Lopes, C. Louise and S.N. Pereira et al., 2017. Geometric morphometrics in mosquitoes: What has been measured? Infect. Genet. Evol., 54: 205-215.

Lorenz, C., T.C. Marques, M.A.M. Sallum and L. Suesdek, 2012. Morphometrical diagnosis of the malaria vectors Anopheles cruzii, An. Homunculus and An. Bellator. Parasites Vectors, 5: 1-7.

Mahendradas, P., 2016. Chikungunya. In: Intraocular Inflammation, Zierhut, M., C. Pavesio, S. Ohno, F. Orefice and N.A. Rao (Eds.). Springer, Berlin, Germany, ISBN:978-3-540-75385-8, pp: 1247-1251.

Rattanarithikul, R., B.A. Harrison, P. Panthusiri and R.E. Coleman, 2005. Illustrated keys to the mosquitoes of Thailand I. background; geographic distribution; lists of genera, subgenera and species and a key to the genera. Southeast Asian J. Trop. Med. Public Health, 36: $1-80$.

Rattanarithikul, R., R.E. Harbach, B.A. Harrison, P. Panthusiri and R.E. Coleman et al., 2010. Illustrated keys to the mosquitoes of Thailand. VI. Tribe Aedini. Southeast Asian J. Trop. Med. Public Health, 41: $1-38$.

Service, M., 2008. Medical Entomology for Students. 4th Edn., Cambridge University Press, Cambridge, UK., ISBN:978-0-521-70928-6, Pages: 299.

Sumruayphol, S., C. Apiwathnasorn, J. Ruangsittichai, P. Sriwichai and S. Attrapadung et al., 2016. DNA barcoding and wing morphometrics to distinguish three Aedes vectors in Thailand. Acta Trop., 159: $1-10$. 
Sungvornyothin, S., R. Kumlert, D.H. Paris, A. Prasartvit and P. Sonthayanon et al., 2019. Geometric morphometrics of the scutum for differentiation of Trombiculid mites within the genus Walchia (Acariformes: Prostigmata: Trombiculidae) a probable vector of scrub typhus. Ticks Tick Borne Dis., 10: 495-503.

Virginio, F., P.O. Vidal and L. Suesdek, 2015. Wing sexual dimorphism of pathogen-vector culicids. Parasites Vectors, 8: 1-9.
Vu, D.M., D. Jungkind and A.D. LaBeaud, 2017. Chikungunya virus. Clinics Lab. Med., 37: 371-382.

WHO., 2007. Anopheline Species Complexes in South and South-East Asia Anopheline Species Complexes in South and South-East Asia. World Health Organization, Geneva, Switzerland, ISBN: 978-92-9022-294-1, Pages: 102.

Wilke, A.B.B., R.D.O. Christe, L.C. Multini, P.O. Vidal and R. Wilk-da-Silva et al., 2016. Morphometric wing characters as a tool for mosquito identification. PloS One, 11: 1-12. 